\title{
Im Universum von (fremden) Welten: Über die Erziehung zum Helden im höfischen Musiktheater des 17. Jahrhunderts
}

\author{
Susanne Rode-Breymann
}

Die fremden Helden mischten sich im 17. Jahrhundert unter all die Heroen, die auf den europäischen Musiktheaterbühnen gezeigt wurden. Die Helden der griechischen Mythologie, die im Zuge der Antikenrezeption ${ }^{1}$ des Humanismus Eingang in das Musiktheater fanden, und die römischen Helden bekamen von den Rändern der Welt her, aus Persien, Afrika, Indien, Konkurrenz.

Der Ausstellungskatalog Götterbilder und Götzendiener in der Frühen Neuzeit. Europas Blick auffremde Religionen ruft das 17. Jahrhundert als Zeit mit einer „nochmals geschärften philologischen, antiquarischen und historischen Kritik und erweiterten

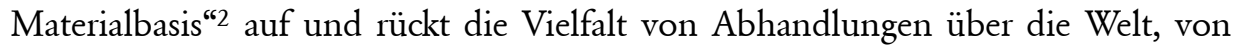
Amerika bis China, in den Blick. „Der Bedarf an [...] ethnographischer und religionsethnographischer Literatur" wie etwa Alter und Neuer Afrikanischer Sitten/ Trachten/Götzendienst/ Grausamkeiten und Landschafften Calender [...] Zum ersten mal mitgetheilet/ durch Simon Kolmayer von Elbingen/ Astrolog. \& Astronom (Nürnberg 1678) sei „offensichtlich sehr groß“ gewesen und habe, so Martin Mulsow, „das

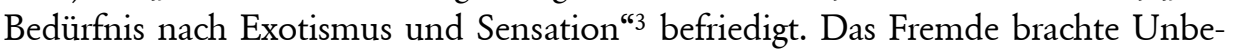
kanntheitsgewinn mit sich und erweiterte die Möglichkeiten auch der Herrscherallegorien im Musiktheater. Dieses Ausgreifen in fremde Welten im Musiktheater ist Thema der nachfolgenden Überlegungen, die - basierend auf der Studie Musiktheater eines Kaiserpaars. Wien 1677-17054 - exemplarisch an den Habsburger Kaiserhof im 17. und beginnenden 18. Jahrhundert führen.

\section{Herrscherallegorien}

Musiktheater stand am Hof von Kaiser Leopold I. im Dienst der Herrschaftslegitimation und zeigte Heroengeschichten, mit denen der eigene Stellenwert heraus-

1 Vgl. dazu Ulrich Heinen (Hg.): Welche Antike? Konkurrierende Rezeption des Altertums im Barock, 2 Bde. (Wolfenbütteler Arbeiten zur Barockforschung; 47). Wiesbaden 2011.

2 Maria Effinger [et al.] (Hg.): Götterbilder und Götzendiener in der Frühen Neuzeit. Europas Blick auf fremde Religionen (Katalog zur Ausstellung vom 15. Februar bis 25. November 2012, Universitätsbibliothek Heidelberg). Heidelberg 2012, 11.

3 Martin Mulsow: Josephe.-F. Lafitau und die Entdeckung der Religions- und Kulturvergleiche. In: Maria Effinger [et al.] (Hg.): Götterbilder und Götzendiener in der Früben Neuzeit. Europas Blick auf fremde Religionen (Katalog zur Ausstellung vom 15. Februar bis 25. November 2012, Universitätsbibliothek Heidelberg). Heidelberg 2012, 37-48, hier 38.

4 Susanne Rode-Breymann: Musiktheater eines Kaiserpaars. Wien 1677-1705. Hildesheim 2010. 
gestellt, und Bilder angemessenen Verhaltens des Herrschers vorgeführt wurden. Um die Weite seines Herrschaftsbereichs vor Augen zu führen, wurde der Herrscher als universal siegreicher Held gezeigt, als ein Held, der in der eigenen Welt wie in fremden Welten (bis hin zur Unterwelt) obsiegt und dessen Machtfülle nicht an den Grenzen seines realen politischen Machtbereiches endet.

Um nur einige Beispiele zu nennen: Der römische Kaiser Hadrian, der römische König Servius Tullius, der syrische König Antiochos, die persischen Könige Kyros und Xerxes, Alexander der Große, Odysseus und Telemachos ${ }^{5}$ - sie alle wurden auf der habsburgischen Opernbühne in den Dienst einer Tugendtypologie ${ }^{6}$ genommen, und dies nicht mit dem Ziel, historische Persönlichkeiten zu zeigen. Vielmehr ging es um „Verkörperungen universalhistorisch gültiger [...] Wahrheiten und Maximen"7. Auf dem Weg der Allegorie wurde so ein Bild von Kaiser Leopold und seinen Herrschertugenden inszeniert und verbreitet, ${ }^{8}$ das heißt ein „Rollenmodell, das er verkörperte“ oder das er „,verkörpern wollte“. ${ }^{9}$ Dem Musiktheater, das „die Tugenden des Herrschers [...] öffentlichkeitswirksam“10 vergegenwärtigte, fiel damit eine zentrale Rolle innerhalb der „Selbstthematisierung" ${ }^{11}$ des Kaisers zu.

Alexander der Große, Eroberer Persiens, war in Wien zwischen La Generosità d'Alessandro (1662) und L'Euleo festeggiante nel Ritorno d'Alessandro Magno dall'Indie (1699) mehrfach Titelfigur im Musiktheater. In La Generosità d'Alessandro wird Alexander als Ebenbild von Leopolds Großmut (generosità) gezeigt. Er gibt „den gefangenen Töchtern des Perserkönigs Darius die Freiheit und diesem sein erober-

5 Zur Schreibweise der Herrschernamen: Wenn im vorliegenden Beitrag von den Musiktheaterwerken am Wiener Hof die Rede ist, werden die Namen dem jeweiligen Libretto folgend verwendet. Am Wiener Hof war es üblich, neben dem italienischen Libretto auch eine deutsche Übersetzung des Librettos zu drucken. Im vorliegenden Text wird aus den deutschen Übersetzungen zitiert.

6 Vgl. Franz Matsche: Die Kunst im Dienste der Staatsidee Kaiser Karls VI. Ikonographie, Ikonologie und Programmatik des „Kaiserstils“, Bd. 1 (Beiträge zur Kunstgeschichte; 16,1). Berlin und New York 1981, 238.

7 Ebd., 240.

8 Vgl. Jutta Schumann: Die andere Sonne. Kaiserbild und Medienstrategien im Zeitalter Leopolds I. (Institut für Europäische Kulturgeschichte der Universität Augsburg, Colloquia Augustana; 17). Berlin 2003, 14.

9 Christoph Kampmann [et al.] (Hg.): Bourbon - Habsburg - Oranien. Konkurrierende Modelle im dynastischen Europa um 1700. Köln [u.a.] 2008, 5.

10 Matsche: Die Kunst im Dienste der Staatsidee (Anm. 6), 57.

11 Volker Kapp: Spielen und Mitspielen. Literatur und höfische Repräsentation zur Zeit Ludwigs XIV. In: Fritz Reckow (Hg.): Die Inszenierung des Absolutismus. Politische Begründung und künstlerische Gestaltung höfischer Feste im Frankreich Ludwigs XIV. (Atzelsberger Gespräche 1990, 5 Vorträge). Erlangen 1992, 105-139, hier 117. Vgl. ähnlich Maria Goloubeva: The Glorification of Emperor Leopold I in Image, Spectacle and Text (Veröffentlichungen des Instituts für Europäische Geschichte Mainz. Abteilung für Universalgeschichte; 184). Mainz 2000, 23: Sie spricht von der Oper „as the favoured genre of the musical, literary and even visual self-expression of Leopold's court". 
tes Reich"12 zurück. 1686, also nach dem triumphalen habsburgischen Sieg gegen die Türken und der Befreiung Wiens 1683, spiegelt Alexander der Große in Il Nodo Gordiano Leopolds neues siegreich-triumphierendes Herrscherimage, das heißt seine „Mission der Führung des Abendlandes im Kampf gegen den [türkischen] Erbfeind“". ${ }^{13}$ Alexander, so wird im Inhalt zusammengefasst,

bemächtigte sich selber: und befliesse sich diesen Knopff loß zu binden. Weil er es aber durch andere Mittel nicht zu weg bringen konte / durchnidte er selben mit seinem Schwerdte; Ist auch folgends / wie Weltkündig / zu einem sehr mächtigen Herrscher und Oberherrn der Welt worden. Er besigte mit höchster Ruhmwürdigkeit die Griechen / Persier / und Indianer / unter denen absonderlich den König Porus / welcher jung / starck und sehr tapffer war. Dieses bezeuget Plutarchus in deß Alexanders Leben. ${ }^{14}$

In der Vorrede an Kaiserin Eleonore Magdalena wird die Allegorie auf Leopold mit folgenden Worten erläutert:

Die Schuld-Pflicht erforderte es / daß der von Alexander durch den Degen entlöste Gordische Knopff an dem glorwürdigsten Geburts-Tag Dero Durchleuchtigsten Gemahlen / Leopold / dessen Helden-Thaten viel ruhmbarer seynd als jene deß Macedonier / zu Befrolockung dienen sollte. Alexander verschenckte Stätt: Dero Großmächtigster Kayser erhaltet anderen die Cronen: Jener stellte die Gebüete zu ruck / dieser macht das bemächtigte wiederumb erstatten: Jener offnete mit dem Degen einen Gordischen Knopff : Dieser entlössete vielfältige Kriegs-Verwirrungen $[\ldots] .^{15}$

In L'Euleo festeggiante nel Ritorno d'Alessandro Magno dall'Indie schließlich feiert Alexander der Große nach seinen Siegen in Indien am Fluss Euleus seine Hochzeit mit Statira, seine Heerführer vermählt er mit Perserinnen. Bei der 1699 zu Ehren des Thronfolgers Joseph I. aufgeführten Geburtstags-Serenata L'Euleo festeggiante wurde an nichts gespart, es wirkten "mindestens 110 Instrumentalisten in zwei Chören“ mit, „davon mindestens 50 Streicher, etwa 40 Bläser und 30 Lauteninstrumente "16 _ verständlicherweise, denn es war eine Aufführung im Freien, am Teich der Favorita, die ein entsprechendes Klangvolumen erforderte.

Die Geschichten der persischen Könige Kyros und Xerxes sind Begebenheiten am Wegesrand der Feldzüge von Alexander: In Creso (1678) etwa kämpft der lydische König Kroisos gegen den persischen König Kyros, von dem er gefangen genommen, zum Tode verurteilt, schließlich jedoch begnadigt wird. Am Ende tan-

12 Herbert Seifert: Die Oper am Wiener Kaiserhof im 17. Jabrhundert (Wiener Veröffentlichungen zur Musikgeschichte; 25). Tutzing 1985, 221.

13 Martin Wrede: Türkenkrieger, Türkensieger. Leopold I. und Ludwig XIV. als Retter und Ritter der Christenheit. In: Christoph Kampmann [et al.] (Hg.): Bourbon - Habsburg - Oranien. Konkurrierende Modelle im dynastischen Europa um 1700. Köln [u.a.] 2008, 149-165, hier 157.

14 Niccolò Minato: Der Gordische Knopff. Libretto, dt. Übersetzung von Carl Ignaz Langetl. Wien: Susanna Christina Cosmerovius 1686 (A-Wn 406.795-B M), ohne Paginierung.

15 Ebd.

16 Seifert: Die Oper am Wiener Kaiserhof (Anm. 12), 362, Anm. 107. 
zen die „Planeten um den Kaiser“17 und führen vor Augen, dass er im „Zentrum der Welt" steht und der „ordnende Mittelpunkt der Welt"18 ist.

Der persische König Kyros ist kein weiser Herrscher, dem Milde wie Kaiser Leopold angeboren ist, sondern er wandelt sich in der Oper vom strafenden, Kroisos zum Tode verurteilenden Herrscher zum begnadigenden Herrscher. Der lydische König Kroisos, Personifikation habsburgischen Verhaltens, muss Milde dagegen nicht erst erwerben. Ihm ist sie angeboren, und sie greift sogar gegenüber Orsane, dessen Reue Kroisos genügt, um ihn trotz Verrats zu begnadigen - basierend auf der Einsicht, dass Milde „bei der Bekämpfung eines Übels wirkungsvoller“ ist „als Gewalt“, dass es „vortrefflicher“ ist, „einem Volk durch Clementia Grund zu freiem Atem zu geben, als durch Tyrannis Gelegenheit zur Verschwörung."19 Clementia steht auch im Zentrum von Temistocle in Persia (1681). Hier ist es Xerxes, der einem Kontrahenten gegenüber Milde walten lässt, der des Verrats an ihm verdächtig ist.

All diese Opern, La Generosità d'Alessandro (1662), Creso (1678), Temistocle in Persia (1681), Il Nodo Gordiano (1686), L'Euleo festeggiante nel Ritorno d'Alessandro Magno dall'Indie (1699), Temistocle (1701), wurden aus Anlass des Geburtstags von Kaiser Leopold I. oder des Geburtstags seines Sohnes Joseph I. aufgeführt: „[A]n denen allerhöchsten Geburths-Tagen derer regierenden Kayserlichen Majestäten" seien, so Johann Basilius Küchelbecker, „etliche unvergleichliche Opern gespielet ${ }^{“ 20}$ worden. Sie sind Teil eines umfangreichen musiktheatralen Korpus von Stücken, die jeweils nur ein Mal, eben zu bestimmten Anlässen, aufgeführt wurden. Im Zeitraum der dritten Ehe von Kaiser Leopold mit Eleonore Magdalena, das heißt zwischen Dezember 1767 und dem Tod Leopolds 1705, wurden mehrere hundert neu komponierte (musiktheatrale) Werke aufgeführt, ${ }^{21}$ davon allein rund 120 szenisch realisierte Werke. Das wurde von einem Produktionsteam bewerkstelligt, anfangs bestehend aus dem Librettisten Niccolò Minato, dem Komponisten Antonio Draghi, dem Bühnenbildner und -archtitekt Lodovico Ottavio Burnacini, dem Tanzmeister Santo Ventura und dem Ballettmusikkomponisten Johann Heinrich Schmelzer. Routiniert brachte dieses Team gemeinsam mit Sängern, Musikern, Tänzern und tanzenden Mitgliedern der kaiserlichen Familie eine Produktion nach der anderen auf die Bühne - rund vierhundert ${ }^{22}$ während Leopolds Regierungszeit.

Die souveräne Handwerklichkeit und institutionelle Konstanz dieses Teams war Grundlage auch für eine spezifische Kommunikationssituation: Libretto, Musik,

17 Rouven Pons: „Wo der gekrönte Löw hat seinen Kayser-Sitz“. Herrschaftsrepräsentation am Wiener Kaiserhof zur Zeit Leopolds I. (Deutsche Hochschulschriften; 1195). Egelsbach [u.a.] 2001, 217. für das Haus Habsburg. Wien 1973, 99.

Veronika Pokorny: Clementia Austriaca. Studien zur besonderen Bedeutung der Clementia Principi

Johann Basilius Küchelbecker: Allerneueste Nachricht vom Römisch-Kayserl. Hofe. Nebst einer ausfübrlichen historischen Beschreibung der Kayserlichen Residentz-Stadt Wien, und der umliegenden Oerter. Hannover 1730, 252.

21 Vgl. Seifert: Die Oper am Wiener Kaiserhof (Anm. 12), 486-582.

22 Elisabeth Hilscher: Mit Leier und Schwert. Die Habsburger und die Musik. Graz [u.a.] 2000, 128. 
Aufführungsort, Bühnenraum und Tanz fungierten im Musiktheatersystem des Wiener Kaiserhofes gleichermaßen als Medien der Kommunikation. Innerhalb dieses ästhetischen Systems sah man sich nicht genötigt, Aussagen mehrfach, durch jede der beteiligten Bühnenkünste kommunikationssicher zu vermitteln. Als kommunikationssicher galt, was entweder durch das Libretto oder die Musik oder den Bühnenraum oder den Tanz vermittelt wurde. Das Gesagte und das Gezeigte standen mithin ebenbürtig im Dienst einer Inszenierung fürstlicher Macht. Das gilt auch für die Darstellung des Fremden: Es war eine Sache der Librettistik, gelegentlich auch eine Sache der Tänze, dies zu verdeutlichen. Die Musik war daran anfangs nicht beteiligt.

\section{Kommunikation des Zeigens}

In den Tänzen der Geburtstagsopern für Kaiser Leopold I. geht es um das Durchmessen des Weltkreises: Die an den Aktschlüssen stehenden Tänze führen nach Rom, Delphi, Sparta, Spanien, Persien, Baktrien, Indien, in das „Mohrenland“. Es tanzen persische Kriegsknechte (etwa in Temistocle [1701]), es tanzen Afrikaner (etwa in Le Fonti della Beotia [1682] oder in Il delizioso ritiro di Lucillo [1698]). Und immer wieder treten ,Mohren' auf: „Mohren / so mit der Madema gekommen“ tanzen am Ende von L'Amare per Virtu (1697) „aus Freud wegen glücklicher Ankunfft in Spanien" oder werden Berenice in der 6. Szene von La Chioma di Berenice (1690) mit den Worten überreicht, „diese gefangenen Mohren“ schicke ihr Ehemann „wegen Seltsamkeit der Gestalt" ${ }^{\text {"23. }}$.

Wenn man genauer auf die Jahreszahlen der Aufführungen schaut, wird ersichtlich, dass es sich um ein Phänomen des Jahrhundertendes handelt. In der zweiten Hälfte der 1660er Jahre, als der habsburgische und der französische Hof auch mit Mitteln der Kunst um ihr höfisches Prestige konkurrierten und mit Hoffesten in allegorischen Wettstreit traten, sah das noch anders aus: Das berühmte, dreitägige Hoffest Les Plaisirs de l'ile enchantée, das Ludwig XIV. 1664 im Schlosspark von Versailles veranstaltet hatte, brachte einen Elefanten und ein Kamel auf die Bühne. Die Habsburger ,antworteten' 1667 mit Rössern, das heißt mit dem Rossballett La Contesa dell'Aria e dell'Acqua: „Leopolds Rossballett“, so beschreibt es Martin Wrede, folgte

ebenso wie Leopolds Rittertum den Regeln zeitgenössischer höfischer Ästhetik und politischer Logik, also nicht zuletzt der des höfischen Konkurrenzkampfes mit Frankreich. Es war eine direkte Erwiderung auf das große Carrousel des cinq nations des Jahres 1662, auf dem Ludwigs XIV. sich nicht nur als römischer Imperator und Universalherrscher präsentiert hatte, sondern vor allem als erster, recht eigentlich einziger Ritter. [...] Beide Feste verkündeten so grundlegende politische Botschaften, die über die bloße höfische Konkurrenz von ,Wien und Versailles' weit hinausgingen. [...] Vor der Hofburg gab es [...] die

23 Niccolò Minato: Die Haare der Berenice. Libretto, dt. Übersetzung. Wien: Susanna Christina Cosmerovius 1690 (A-Wn 25.623-B). 
Antwort: Noch mehr Prachtentfaltung, noch mehr Teilnehmer, Pferde, ,Maschinen', vor allem aber ein Kaiser, der nicht nur als glanzvolles Individuum auftrat - sondern als Vertreter der dynastischen Kontinuität. ${ }^{24}$

Wie sehr bei solchen Festen und insbesondere bei den Opern zu den Namenstagen des habsburgischen Herrscherpaares der Schauwert und das Zeigen im Vordergrund standen, verdeutlichen zum Beispiel die Worte des hessisch-darmstädtischen Gesandten Justus-Eberhard Passer über Le Fonti della Beotia (1682): „[I]n dem grßen Lustgarten zu Schönbrunn“, so berichtet er, seien „auf einem grünen gleichen Platz, Zwischen grünen Spallier Vnd Bindwerken, hohe Berge“ aufgerichtet worden, „darauf Häußer gebaut, in der mitten gantz offen, Vff Beeden seiten 2. frische Brunnquellen“. Ein Höhepunkt des Ganzen seien schließlich die „Bähren Vnd Löwen“ gewesen, die im Interludium „aus den Wäldern“25 gekommen seien.

Der Löwe, „ein vierfüßiges Thier, groß und dick, wild und grausam, von erschrecklichem Anblick“, wie in Johann Heinrich Zedlers Universal-Lexicon zu lesen, werde „wegen seiner edelmüthigen Freudigkeit, tapffern Stärcke und Hertzhafftigkeit, auch unerschrockenem Gemüthe, der König aller wilden Thiere genannt“ und lebe in „Asien, Africa, Persien, und dergleichen warme Länder“. Weiter beschreibt er, dass Löwen „zahm erzogen und übers Meer zu uns nach Europa an Königliche und Fürstliche Höfe überbracht“, dort „theuer verkaufft“ und „theils

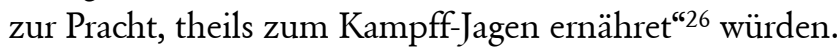

Dieses Wissen über das Tier aus fremden Ländern ging in die zu Eleonore Magdalenas Namenstag aufgeführte „Introduttione d'un balletto“ La Vittoria della Fortezza (1687) ein. Darin stehen Alexander der Große und Lysimachus einander gegenüber. Lysimachus, „deß Agathocles Sohn / einer der vornehmsten auß Macedonien“, der „denen Wissenschaften / fürnehmlich aber der Welt-Weißheit sehr ergeben" ${ }^{27}$ war,

hörte vielmahls deß Weltweißen / Callisthenes Sittenlehre an / und war ihme solcher gestalt geneigt / daß er selbigem / als er in deß Alexanders Ungand fiele / und in Ketten gefangen gehalten wurde / Hülff leistete / und ihn der Feßlen befreyte. Worüber Alexander erzörnend / alsobald verordnete / daß Lysimachus von einem Löwen zerrissen werde. Dem zufolg führte man ihn dem erwildten Thiere vor / und nach deme er sich gegen diesen gantz kühnmüthig genäheret / umbwickelte er den rechten Armen mit seinem Uberrock / stoßte selben den Löwen in den Rachen / und risse ihm die Zunge herauß / erledigte sich auch solcher gestalten der vorstehenden Gefahr. Dessentwegen er dann nochmahls von Alexander nicht minder seiner fürtrefflichen Tugend als verwunderbahren Stärcke halben sehr geliebet und werth gehalten wurde. ${ }^{28}$

24 Wrede: Türkenkrieger, Türkensieger (Anm. 13), 154-155. Justus-Eberhard Passer an die Landgräfin Elisabeth Dorothea von Hessen-Darmstadt, 30. Juli 1682, zitiert nach Seifert: Die Oper am Wiener Kaiserhof (Anm. 12), 788.

26 Johann Heinrich Zedler: Grosses vollständiges Universal-Lexicon aller Wissenschaften und Künste, Bd. 18. Halle und Leipzig 1738, 216-218.

27 Niccolò Minato: Sieg der Stärcke. Libretto, dt. Übersetzung. Wien: Susanna Christina Cosmerovius 1687 (A-Wst A 24.376), ohne Paginierung. 
Das wurde auf der Bellaria an einem „Lust-Orth vor der Stadt in Macedonien“ gezeigt, „nechst an einem Wald: mit etlichen alten Klüfft-Gebäuden; und mit einem verschlossenen Gebäu / worinnen die Löwen verwahret seynd“, und von Löwenwärtern bevölkert, die (in einem Tanz) die Löwen bändigten, bis Alexander dann in der 14. Szene befiehlt, man möge „nicht länger“ verweilen, sondern „Lysimachus dem aller ergrimmsten von" seinen Löwen gegenüberstellen, und er werde sich ,auch selbsten mit Zusehung dieses Kampffes ergötzen“. ${ }^{29}$ Löwenführer bringen den Löwen in der 15. Szene herbei - der Kampf kann beginnen, und wie könnte die überlegene Stärke von Lysimachus (in dem Leopold zu erkennen ist) besser ins Bild gesetzt werden als mit einem solchen Kampf gegen einen Löwen, den Lysimachus in der 17. Szene tötet. Entsprechend beeindruckt ist denn auch Alexander, dessen Macht- und Überlegenheitsanspruch angesichts einer solchen Tat zerrinnt.

Dieser Gattungstyp unter den Namenstagopern zielte auf visuelle Vermittlung von (Herrscher und Herrscherin rühmenden) Sinnbildern. Das Zeigen war wichtiger als das Sagen. In einer Kultur, in der Emblematik ein fest verankerter Wissensbestand war, konnte damit gerechnet werden, dass Kommunikation über Bilder auf dem Musiktheater sicher funktionierte.

\section{Indien}

Kaiserin Eleonore Magdalena brachte zehn Kinder zur Welt, beginnend mit Joseph I., dessen Geburt 1678 mit La Monarchia latina trionfante im Hoftheater auf der Cortina gefeiert wurde, einer "aufwändigen Maschinenoper"30, deren Opulenz Ausdruck der Erleichterung über die Thronfolgergeburt nach zwanzigjähriger Regentschaft von Leopold I. ist. Die staatspolitische Programmatik dieser Festa musica$l e$ ist offenkundig und wurde durch neun Kupferstiche im Libretto eindrucksvoll visualisiert: Joseph I. muss nicht mehr wie sein Vater Leopold I. mit Rössern gegen die Türken kämpfen (Abb. 1), sondern mit seinem Erscheinen beginnt ein neues Zeitalter. Der Vorhang öffnet sich für einen Helden (Abb. 2), dem kräftigere Tiere vom Ende der Welt zu Gebote stehen, mit denen er die Welt erobern kann. Daran lässt die Handlung von La Monarchia latina trionfante keinen Zweifel: Es geht um den Kampf zwischen den vier Weltreichen, personifiziert durch Ninos (den Herrscher der Assyrer), Dareios (den Herrscher der Perser), Alexander (den Herrscher der Griechen) und Caesar (den Herrscher der Römer). Der Sieg und damit die Vorherrschaft gehen letztlich an Caesar, den vielbeschworenen Ahnen der Kaiser des Heiligen Römischen Reiches Deutscher Nation - also an Joseph I.

29 Ebd.

30 Michael Ritter: „Man sieht der Sternen König glantzen“. Der Kaiserhof im barocken Wien als Zentrum deutsch-italienischer Literaturbestrebungen (1653 bis 1718) am besonderen Beispiel der Libretto-Dichtung. Wien 1999, 97. 


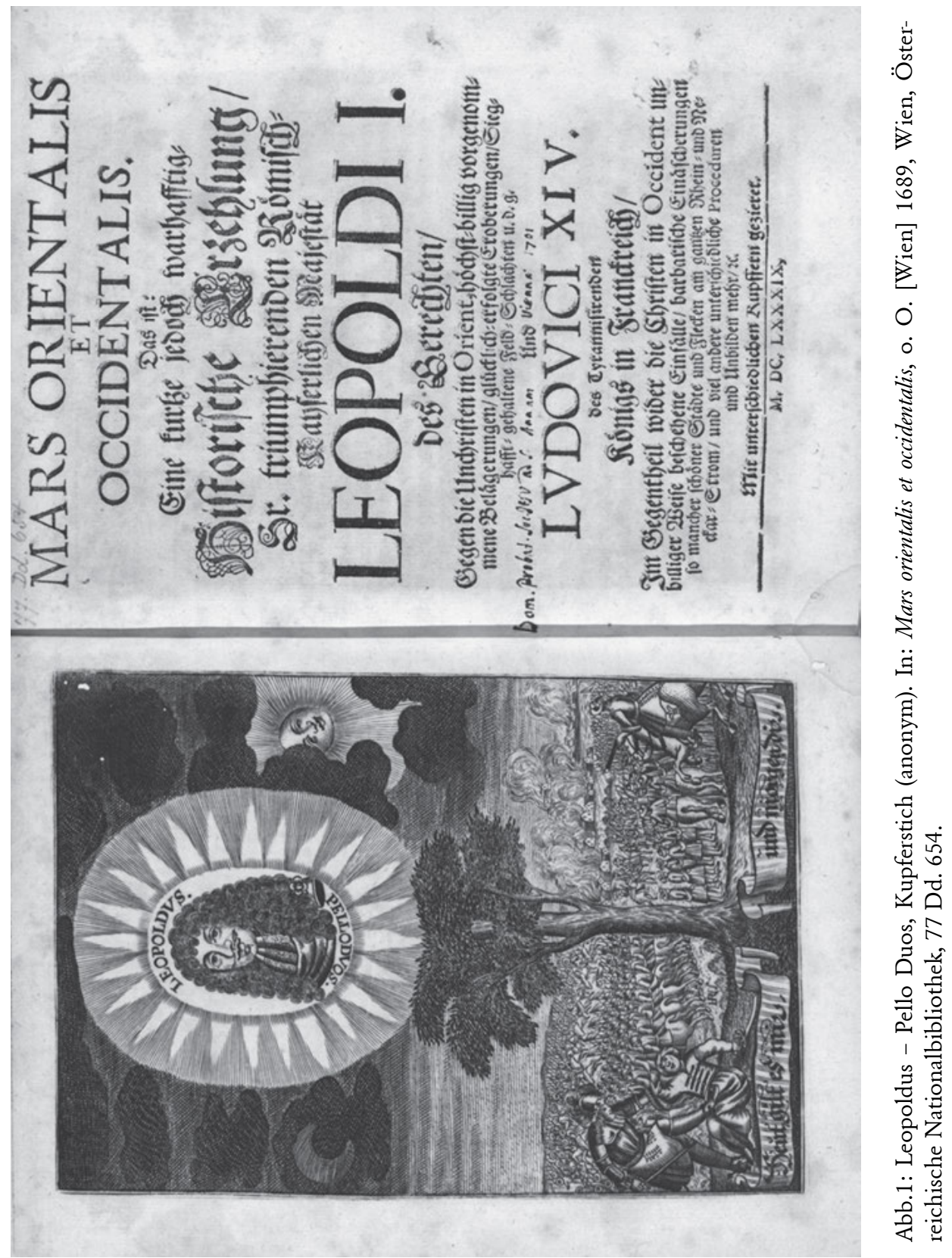




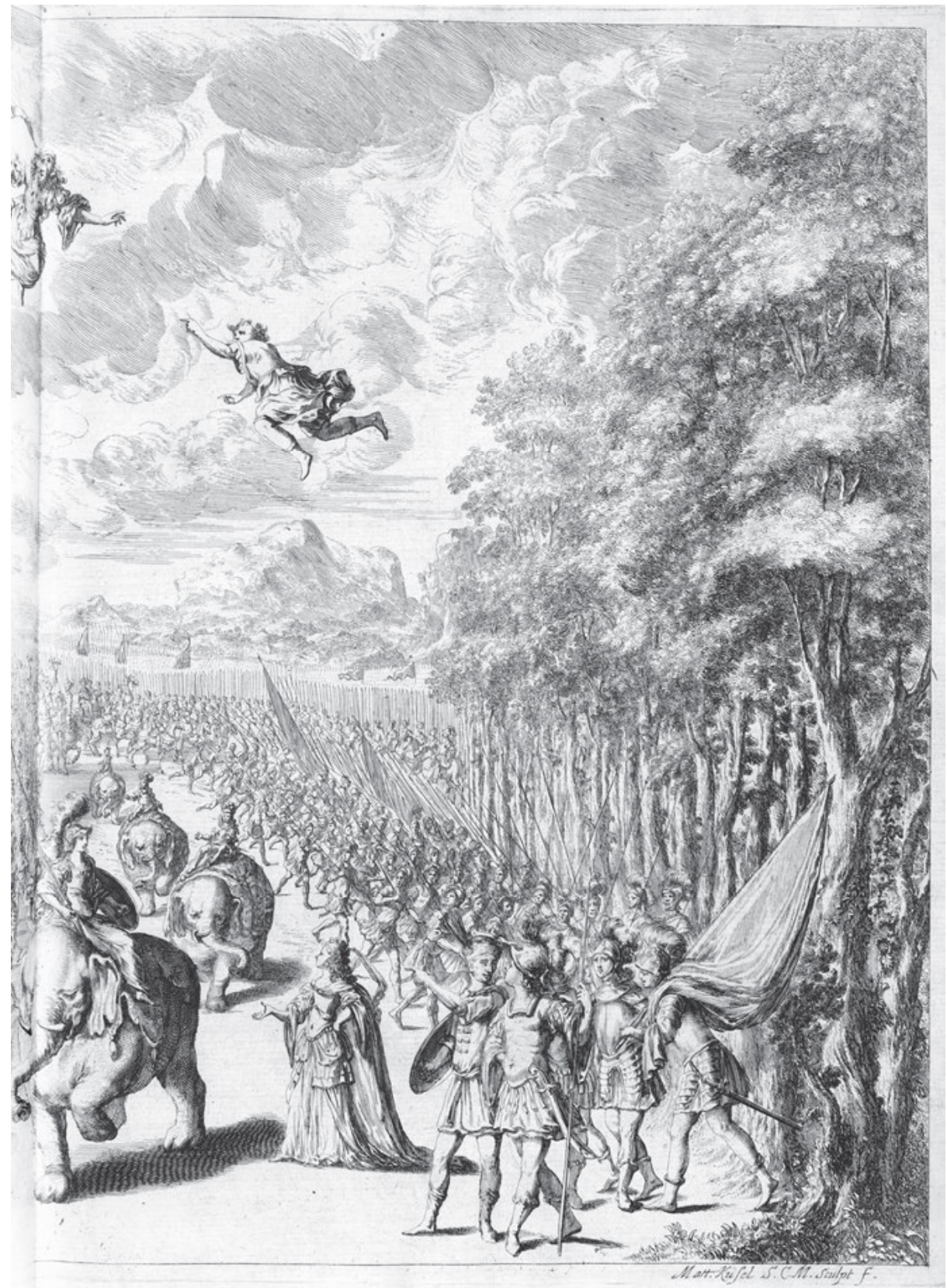

Abb. 2: Elefanten, Kupferstich von Matthäus Küsel. In: Niccolò Minato (Text) und Antonio Draghi (Musik): Die Sig-prangende Römische Monarchey [...]. Wien 1678, Wolfenbüttel, Herzog August Bibliothek, Hn 4 13, http://diglib.hab.de/drucke/hn-4f-13/start.htm, 20. Mai 2016. 
,Der Elefant ist [...] das beträchtlichste Geschöpf dieser Welt', - so hebt Buffon das Elefantenkapitel seiner Naturgeschichte an [...]: ,Er übertrifft alle Lebewesen der Erde an Größe und kommt an Klugheit dem Menschen nahe [... $]^{6}$. In Buffons klassischer Darstellung erstrahlt der Elefant noch einmal in der vollen Glorie von Größe und Weisheit, mit der abendländische Betrachtung ihn umgeben hat, seit er zu Alexanders und Aristoteles' Zeiten imponierend, feierlich und gewandt in ihren Gesichtskreis trat [...]. Der Chorus der Alten ist sich über die einzigartige Intelligenz und Vernünftigkeit des Elefanten einig, und die erbaulich-reizenden Geschichten, die Plinius und Plutarch, Aelian und Oppian von ihm berichten, haben das Entzücken des Abendlandes gebildet, solange es klassisch gebildet war. ${ }^{31}$

Heinrich Zimmer, der große Indienforscher, von dem diese Zeilen stammen, verweist in seiner Natur-, Kultur- und Bewusstseinsgeschichte Spiel um den Elefanten. Ein Buch von indischer Natur auf Georg Christoph von Hartenfels Elephantographia Curiosa (1714): Sie habe

antiken Quellen vertrauend, über den Elefanten mehr Material zur ,Geschichte seines Ruhms` zusammengetragen, als daß sie seine Wirklichkeit erfaßte [...]. Denn seine einzigartige Gestalt, sein bedeutendes Wesen wahrte dem Elefanten die Wunderaura von Ferne und Fabel, auch als er der alten Welt seit Alexanders Zeit vertraute Erscheinung ward: - mitkämpfend in den Heeren der Diadochen, in den Schlachten Pyrrhus' und der Karthager, würdig einherschreitend im Staatspomp der Ptolemäer und den Festzügen der Römer. $^{32}$

Von Hartenfels erwähnt, der Elefant sei „in Deutschland äußerst selten“ und er habe „nur einen einzigen zu Gesicht bekommen“, was ihm aber „an Fülle der Anschauung versagt blieb, besaß er an Buchwissen“, denn er hatte „die antike Literatur durchgepflügt nach Stellen, wo sie den Elefanten erwähnt." "33

Dieser indische ,Held' trat 1678 mit La Monarchia latina trionfante in die habsburgische (Bühnen-)Welt - auch hier als Buchwissen im Kontext einer umfassenden Auseinandersetzung mit antiker Literatur, die deutliche Spuren im Musiktheater hinterließ: Nach zwei 1686 aufgeführten Opern, die das einsetzende PlutarchInteresse des Wiener Hofes dokumentieren, griffen zwischen 1695 und 1699 sechs Opern auf Plutarch zurück, gipfelnd in einer Plutarch-Opern-Trias 1699. Im Zuge dessen kam es zu einer Wiederbegegnung mit dem Elefanten: La Magnanimità di Marco Fabrizio, 1695 zum Namenstag von Leopold I. aufgeführt, geht auf Plutarchs Parallelbiographien Pyrrhos und Marius zurück, und zwar auf die Kapitel 13 bis 21, in denen es um den Krieg zwischen Römern und Tarentinern geht. Zu Pyrrhos' Leuten gehört Kineas, ein Gesandter von großer Klugheit. Nach erfolglosem Verhandeln mit dem Gegner wird er mit dem Bescheid zu Pyrrhos zurückgeschickt, er solle seine Truppen abziehen, sonst werde man weiterkämpfen. Im Gegenzug schicken die Feinde nun ihrerseits einen Gesandten, der mit Pyrrhos

31 Heinrich Zimmer: Spiel um den Elefanten. Ein Buch von indischer Natur. Frankfurt am Main 1979, 19.

32 Ebd., 22.

33 Ebd., 21. 
über die Freigabe von Gefangenen verhandeln soll. Es ist Marcus Fabricius, ein durch seine Kriegserfahrung hoch angesehener Mann: Er ist absolut unbestechlich (und nimmt trotz seiner Armut das von Pyrrhos angebotene Goldgeschenk nicht an), und er ist vollkommen furchtlos: Pyrrhos konfrontiert ihn, der noch nie einen Elefanten gesehen hat, mit dem größten seiner Elefanten. Fabricius aber bewahrt die Ruhe. Darin erkennen Pyrrhos und Kineas die Ebenbürtigkeit dieses Mannes und treten in philosophische Gespräche mit ihm ein, in denen Pyrrhos'

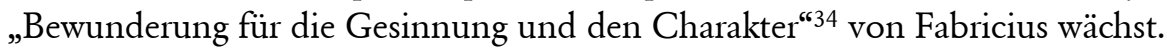

Die Dramatisierung im Libretto von Donato Cupeda schiebt Motive übereinander, Personen (Pyrrhos und Kineas) ineinander, verteilt die Handlungen auf andere Personen, aber die Szene mit dem erschreckend großen Tier findet - allerdings völlig verwandelt - Eingang in das Libretto: Der Diener Lusco hat das Gold, das ihm Pyrrhos gab, nicht weisungsgemäß an Clelia weitergegeben, sondern vergraben. Als ihm Fabricius auf die Schliche zu kommen droht, versucht Lusco ihn mit Hinweis auf ein großes Tier von der Stelle zu verjagen und sagt: „Herr / begib dich doch hinweg; Ich hab erst hier ein wild- und ungeheueres Thier gesehen / es war länger und dicker / als du. Ich weiß nicht / war es ein Löw / oder ein Elephant. Fühle nur / fühle / wie mir das Hertz noch schlagt." ${ }^{\text {"35 }}$

\section{Afrika}

Afrika war in der Frühen Neuzeit ein ,in geographischen Abschnitten“ bekannter Kontinent, der „im traditionellen Weltbild“ zwar fest verortet war, „dessen Erstreckung südlich der Sahara“ jedoch „erst mit den portugiesischen Entdeckungsfahrten des 15. Jahrhunderts in das europäische Bewusstsein rückte und [...] als eine besonders fremdartige Weltzone empfunden wurde“ ${ }^{36}$ Noch im 17. Jahrhundert war Afrika als „geographischer Raum [...] Projektionsfläche für Phantasien des Anderen, des Fremden und Nichtidentischen“ ${ }^{\text {" }} 7$ und blieb „lange ein visuelles Amalgam aus exotischen Tierarten, monsterhaften Fischsorten, und abenteuerlichen Flussläufen".38

Petra Feuerstein-Herz hat die im „Zuge dieser Entdeckungsreisen“ entstandenen kosmografischen Weltbeschreibungen Afrikas am Beispiel der Herzoglichen Bibliothek zu Wolfenbüttel, das heißt anhand von „Reise- und Missionsberich-

34 Ebd., 33.

35 Donato Cupeda: Die Großmüthigkeit Deß Marcus Fabricius. Libretto, dt. Übersetzung. Wien: Susanna Christina Cosmerovius 1695 (A-Wn 407.380-A M), 58.

36 Petra Feuerstein-Herz: Afrika in Wolfenbüttel - Zur globalen Kulturwahrnehmung. In: Claudia Brinker-von der Heyde [et al.] (Hg.): Frühneuzeitliche Bibliotheken als Zentren des europäischen Kulturtransfers. Stuttgart 2014, 177-198, hier 180.

37 Bernhard Klein: Randfiguren. Othello, Oroonoko und die kartographische Repräsentation Afrikas. In: Ina Schabert und Michaela Boenke (Hg.): Imaginationen des Anderen im 16. und 17. Jabrhundert (Wolfenbütteler Forschungen; 97). Wiesbaden 2002, 185-216, hier 185.

Ebd., 200. 
te[n], Landeskunden und Topographien über den afrikanischen Kontinent" ${ }^{\star 39}$, untersucht und stützt sich dabei auf Marilia dos Santos Lopes Untersuchung zur Rezeption des Wissens über Afrika auf dem deutschen Buchmarkt im 16. und 17. Jabrbundert. ${ }^{40}$ Bedenke man, so Petra Feuerstein-Herz, „dass über den afrikanischen Kontinent [...] eine vergleichsweise eher geringe Zahl von Büchern publiziert" worden sei, erstaune es, „in welcher Breite [Herzog] August die entsprechende Literatur gesammelt" habe. „Bei diesen Werken“ handele es sich

überwiegend um Berichte von Pilger- und Missionsreisen, Kolonialschrifttum und Reisebeschreibungen, auch um einzelne topographische Werke. Neben den [...] frühen Zeugnissen des Leo Africanus und anderer scheint August kontinuierlich die Publikationen über den afrikanischen Kontinent verfolgt zu haben. ${ }^{41}$

Leider fehlt eine entsprechende Studie über die Büchersammlung von Kaiser Leopold I. Sicher waren aber auch dort die Karten des afrikanischen Kontinents bekannt, anhand derer Bernhard Klein im Band Imaginationen des Anderen im 16. und 17. Jahrbundert ${ }^{42}$ den Wandel des europäischen Bildes von Afrika beschrieben hat bis hin zur „wahrscheinlich erfolgreichste[n] Afrikakarte des 17. Jahrhunderts“ von Willem Janszoon Blaeu, die nach „1617 in zahlreichen Auflagen gedruckt" ${ }^{\star 43}$ wurde (Abb. 3). Darauf ist der Kontinent kartografisch und zugleich durch Menschen repräsentiert, die an den beiden Seiten der Karte klassifizierend angeordnet sind: „Eine klare Farbhierarchie platziert weiße Nordafrikaner an erster Stelle, nach unten nehmen die Figuren an Dunkelheit und Nacktheit zu." ${ }^{\text {"44 }}$

Der 1697 zum Geburtstag von Eleonora Magdalena aufgeführte Einakter Le Piramidi d'Egitto führt in den Nordosten des afrikanischen Kontinents. Das Stück spielt auf einem Platz in Memphis, darauf (laut Libretto zwei, nach dem Kupferstich im Libretto) eine Pyramide, auf der Bilder und Zeichen zu sehen sind (Abb. 4). Das Stück kommt nahezu ohne Handlung aus: Es belehrt als Dialog des Königs von Ägypten mit je drei vornehmen Herren und Damen aus Ägypten, erschließt emblematische Wissensbestände ${ }^{45}$ und zeigt die Anwendung dieses Wissens. ${ }^{46}$

39 Feuerstein-Herz: Afrika in Wolfenbüttel (Anm. 36), 180.

40 Ebd., 182. Marilia dos Santos Lopes: Afrika. Eine neue Welt in deutschen Schriften des 16. und 17. Jahrbunderts (Beiträge zur Kolonial- und Überseegeschichte; 53). Stuttgart 1992.

41 Feuerstein-Herz: Afrika in Wolfenbüttel (Anm. 36), 187-188.

42 Vgl. Klein: Randfiguren (Anm. 37).

43 Ebd., 207.

44 Ebd.

45 Vgl. dazu Reinhard Strohm: „Costanza e Fortezza“: Investigation of the Baroque Ideology. In: Daniela Gallingani (Hg.): I Bibiena. Una famiglia in scena: da Bologna all'Europa (Saggi e documenti; 188). Florenz 2002, 75-92, hier 76: „The passionate sentences with which Michel Foucault describes - or rather deplores - the shift in cultural attitudes from the sixteenth to the seventeenth century in Europe, seem to make the same point in two distinct domains (although they are strung together in a single rhetorical climax): one in the domain of the senses, the other in that of knowledge, memory and learning."

46 Vgl. dazu Ritter: „Man siebt der Sternen König glantzen“(Anm. 30), 185-203. 


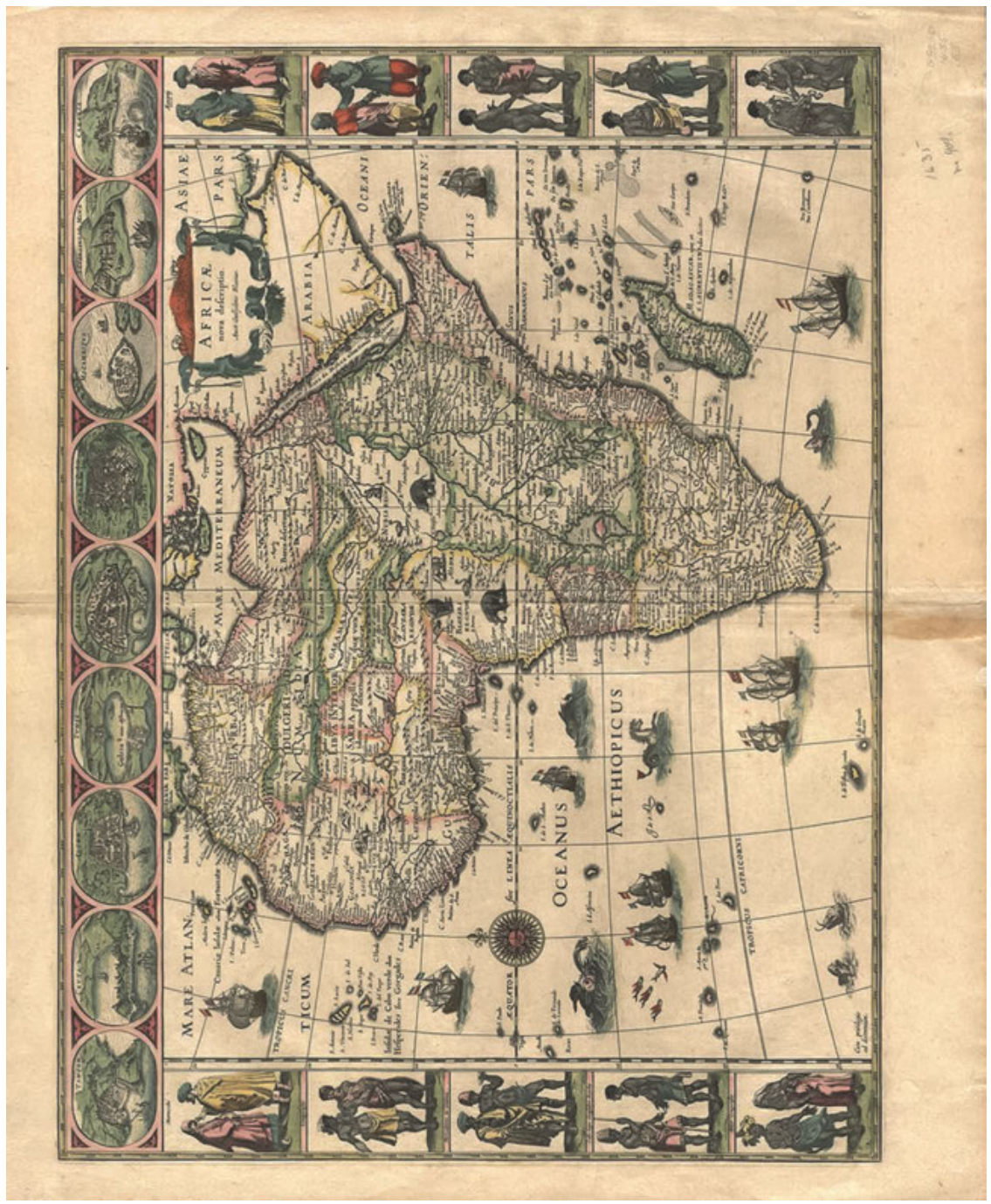

焉

i

촌 응

之े

촌

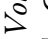

ชั

离

胥

. จ :

$3 \cdot$

5.

50

$\circlearrowleft$

$\ddot{\exists} \Xi$

ज़

离

ีㅗㅇ

$\ddot{\Xi}$

ชं

昰

$\ddot{3}$

ำ

동.

$\ddot{m} \cup$

ㅇํㄴ 
Spilen in fo dufftrer Rituffee Saeiffet twol im Sdzatten pilen.

zere,

Dafi im finffert man ftets ppilet) Jeoer Gey fid felbit befindet/ Das Epil alles sied̨t ver Øútet, Znd fver fpilt / ift offte erblindet.

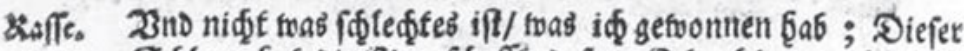

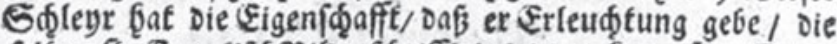

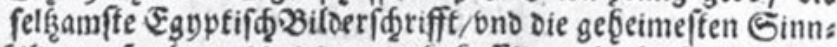

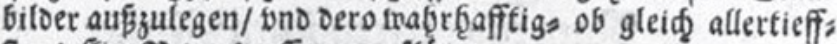
fimnigften Bedeutnuffen zu erfláren.

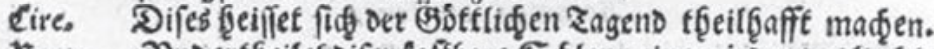

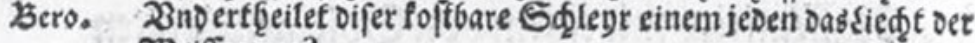
2Beiffagung?

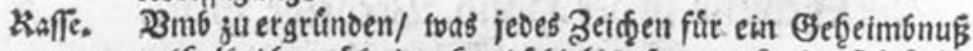
vecor. \{

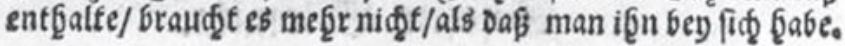
23underliфie Figenfdaffit , felzame Sabe !

Gero.

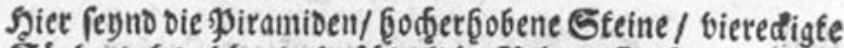

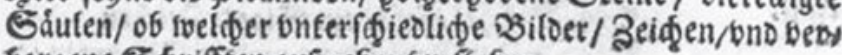

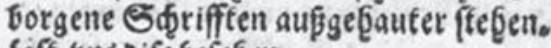

Cire. Sajt bus dife beję̧ent.

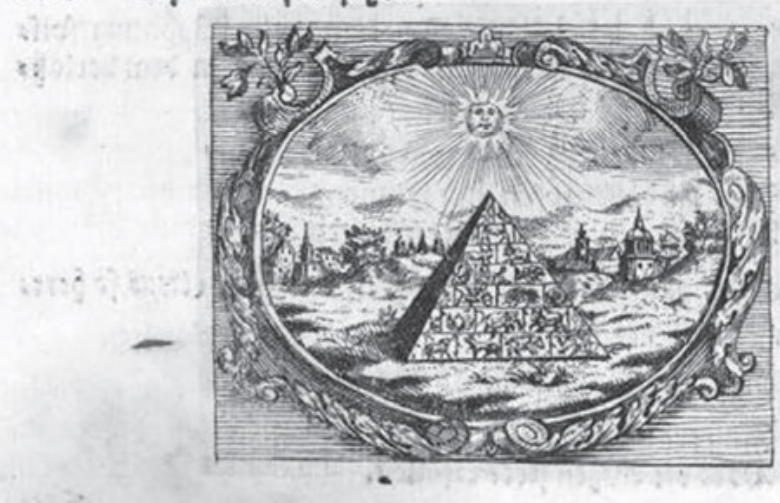

Abb. 4: Pyramide mit Hieroglyphen, Holzschnitt. In: Niccolò Minato (Text) und Antonio Draghi (Musik): Die Egyptischen Piramiden Oder Feuer-Säulen [...]. Wien 1697, Fol. A 4v, Wolfenbüttel, Herzog August Bibliothek, http://diglib.hab.de/drucke/xb-8671/start.htm, 20. Mai 2016. 
König Rassemitico reicht reihum jedem den goldgewirkten Schleier der Proserpina, den er in „deß Pluto Schatten-volle[r] Wohnung" errungen hat und der die Eigenschaft hat, „Erleuchtung [...] für die seltsame Egyptisch[e] Bilderschrifft“ zu geben ,und die geheimesten Sinnbilder außzulegen / und dero wahrhafftig- ob gleich allertieffsinnigsten Bedeutnussen zu erklären" ${ }^{4}{ }^{47}$ Erst kommen die drei Männer an die Reihe, unter Wirkung des Schleiers Bedeutungen erklären zu können. Das macht die Frauen nervös. Sie fühlen sich zurückgesetzt: „So setzet man uns noch den Männern nach?" ${ }^{48}$ wenden sie gemeinsam ein und verkünden Unwillen und Zorn darüber (schließlich ist dieses ,kluge` Bühnenstück eine Verbeugung vor der klugen Kaiserin). Dann ist die Reihe an den drei Frauen, aber nun ist es mit der Einhelligkeit nicht weit her, vielmehr müssen sie zunächst eine Regelung finden, wer von ihnen (die Schönste, die Älteste) zuerst zum Zuge kommen soll.

Abgesehen von diesem rudimentären Handlungsgerüst folgt die Dramaturgie des Stücks dem Prinzip des Wissenszuwachses: Unter der Wirkung des Schleiers zählt jede Person zunächst emblematisches Grundwissen auf. Als zum Beispiel Cirene endlich unter die Erleuchtung des Schleiers kommt, sieht sie gleich eine ganze Reihe von Sinnbildern vor dem inneren Auge:

Der Schwan ist ein Sinnbild der Reinigkeit: Der Biber stellet vor die Liebe deß Lebens: und die Vorbildung deß jenigen / welcher seinen eignen Nutzen ohne deß Nächsten Schaden suchet / ist die Biene / welche von denen Blumen saugt / und sie doch nicht verderbet. [...] So lang das Crocodill lebt / so lang wachst es: Von eben so unersättlicher Art ist der Ehrgeitz / indem er immerdar zuenimt. ${ }^{49}$

Dann erläutert jede Person ein (im Libretto abgedrucktes) Emblem (Abb. 5) und überträgt dessen Bedeutung auf höfische Gegebenheiten, als werde die Fähigkeit zum Transfer des Grundwissens geprüft. Sesostre etwa muss die im Gesicht einem Hund gleichende Gottheit Anubis als Sinnbild der Treue entschlüsseln:

Der Hund bellet die Feinde an / und schmeichlet den Freunden. Disem folge ein jeder Herrscher nach. Wer wol dienet / werde belohnt / wer übel dienet / weggejagt. Der Hund widersetzet sich durch sein Bellen denen Nachstellungen / und erweckt den jenigen / dem man nachstellet. Dises thue auch jeder vornehmer Hof-Bedienter: Wer übel handlet / dem widerseze er sich; und dem / der schlafft / erwecke er. Der Hund bewahret die Herde vor dem Wolff / und andern wilden Thieren : Der Fürst lasse gleichfals nicht zu / daß der Unschuldige denen Meineydigen Nachstellungen außgesetzet seye / und daß das unschuldige Lamb von den wilden Thieren zerrissen werde..$^{50}$

47 Niccolò Minato: Die Egyptischen Piramiden Oder Feuer-Säulen. Libretto, dt. Übersetzung. Wien 1697 (A-Wn 406.739-B M), ohne Paginierung, http://diglib.hab.de/drucke/xb-8671/start.htm, 3. Februar 2016.

Ebd.

49 Ebd.

50 Ebd. 


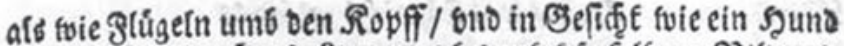

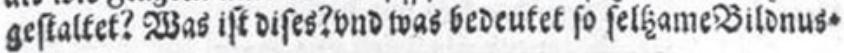

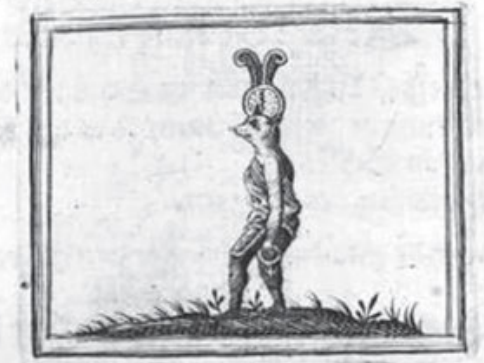

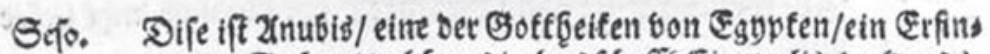

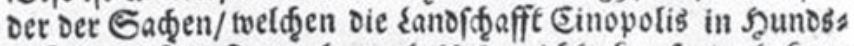

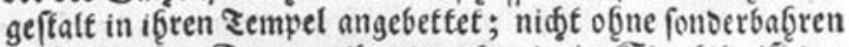
Geţeimmus : Dann tveilen oer Şund ein Sinnbilo ift Der zreue/ vno bey difen verfef̧rt: vno zugenoarmen Beiten jels fen eine auffreḑfe rreue gefunden wiro / po veroient tvol oer jenige/ Der mit Der Zreute bezief̧rt ift / onter die Gstfer geffellt

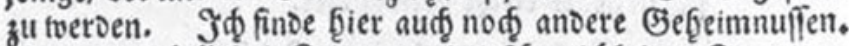

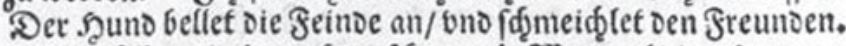

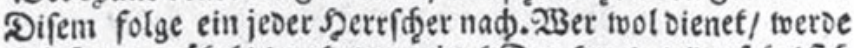

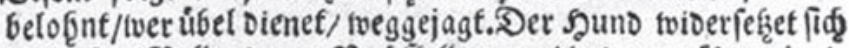
outh fein Bellen oenen गradjifellungen / bno ertvedt oen jenis

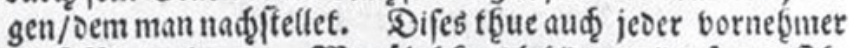

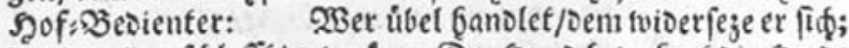

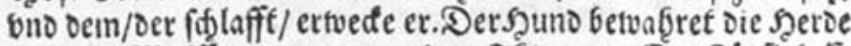

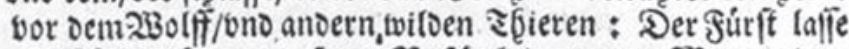

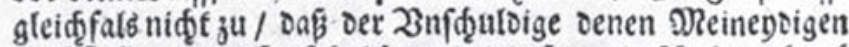

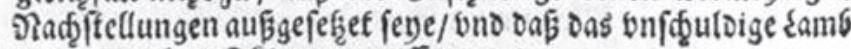
bon Den fvilden ₹b̨ieten jerriffen tverde.

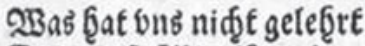

Das tveife 2lltert6um!

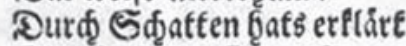

Det ग?ad̨twelt Eigent6̧um.

Cire.

Abb. 5: Anubis, Holzschnitt. In: Niccolò Minato (Text) und Antonio Draghi (Musik): Die Egyptischen Piramiden Oder Feuer-Säulen [...]. Wien 1697, Fol. B 3ㅁ, Wolfenbüttel, Herzog August Bibliothek, http://diglib.hab.de/drucke/xb-8671/start.htm, 20. Mai 2016. 
Die Männer entdecken unter der Wirkung des Schleiers die Geheimnisse von Vaterlandsliebe, Treue und Gleichmaß der Weltordnung, die Frauen werden bezüglich anderer Themen hellsichtig: Es geht um Treue, Standhaftigkeit, Gradlinigkeit und Nachfolge.

Musiktheater arbeitete, wie dieses Beispiel verdeutlicht, dem höfischen Wissenserwerb über die Fremde zu: Gewährsmann für Ägypten war am Habsburger Kaiserhof Athanasius Kircher, der bekannteste Ägyptenforscher des 17. Jahrhunderts. In ihrer Studie Welterkenntnis aus Musik. Athanasius Kirchers „Musurgia universalis“ und die Universalwissenschaft im 17. Jabrbundert hat Melanie Wald die Musurgia universalis als Fürstenspiegel beschrieben und auf deren „enge Bindung [...] an den die Musik liebenden und selbst ausübenden Kaiser Ferdinand III. und dessen Bruder Leopold Wilhelm“ hingewiesen: Das, was Kircher „,von der Musik erkennt und darstellt", solle, so Wald,

eine breite Wirkung gerade bei denen gewinnen, die [...] ihre Prinzipien durch eigene Handlungen jenseits der reinen Musikausübung, nämlich gesellschaftlich umzusetzen vermögen. Damit stellt sich die Musurgia letztlich [...] als ein Fürstenspiegel dar und als die ideelle Begründung eines Friedens, der nicht als Unterdrückung jeden Dissenses imaginiert wird, sondern als Harmonie aufgrund von Verschiedenheiten. ${ }^{51}$

Aufgerufen wird dies schon in der Widmung der Musurgia universalis an Erzherzog Leopold Wilhelm, also den Onkel von Leopold I. Ein Blatt aus dem Turris Babel und ein Blatt aus dem Opernlibretto von Le Piramidi d'Egitto (Abb. 6a und b) machen augenfällig, woher das Wissen über Ägypten ${ }^{52}$ am Wiener Hof kam.

Die fremde afrikanische Welt wird auch in der Festa per Musica namens Il ritorno di Giulio Cesare Vincitore della Mauretania von Giovanni Bononcini und Donato Cupeda aufgerufen, ${ }^{53}$ die aus Anlass der glücklichen Rückkehr des Thronfolgers Joseph I. nach der Einnahme von Landau in der Rheinpfalz entstand und im Dezember 1704 aufgeführt wurde.

Gut platziert in der Mitte der Festa werden Julius Caesar, der Mauretanien unterworfen hat, und König Iuba II., „Ré d’Africa“, den Caesar dort gefangen genommen hat, einander gegenübergestellt. Die Szene zeigt die gewaltige kriegerische Überlegenheit Caesars und steigert das Gefälle zwischen Sieger und Besiegtem drastisch gegenüber den bisherigen Gepflogenheiten im Wiener Musiktheater, in

51 Melanie Wald: Welterkenntnis aus Musik. Athanasius Kirchers „Musurgia universalis“ und die Universalwissenschaft im 17. Jahrhundert (Schweizer Beiträge zur Musikforschung; 4). Kassel [u.a.] 2006, 180.

52 Vgl. dazu Melanie Ulz: Isis - Maria - Puza. Ägyptenfaszination und Kulturvergleich. In: Maria Effinger [et al.] (Hg.): Götterbilder und Götzendiener in der Frühen Neuzeit. Europas Blick auf fremde Religionen (Katalog zur Ausstellung vom 15. Februar bis 25. November 2012, Universitätsbibliothek Heidelberg). Heidelberg 2012, 59-68.

53 Donato Cupeda: Il ritorno di Giulio Cesare. Libretto, dt. Übersetzung von Liesel B. Sayre. In: Susanne Rode-Breymann und Heike Sauer (Hg.): Programmbeft zur Auffübrung in der Hochschule für Musik Köln. Köln 2003, 18-30. Giovanni Bonocini: Il ritorno di Giulio Cesare. Partitur. (A-Wn. Mus. Hs. 16.019) 


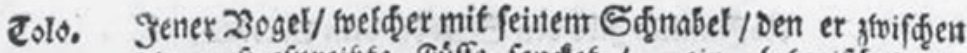

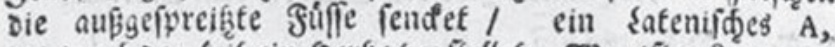
bno ourd oen seib ein Jertze borffellet; Wer iff er? yno was foll ex beoeuten?

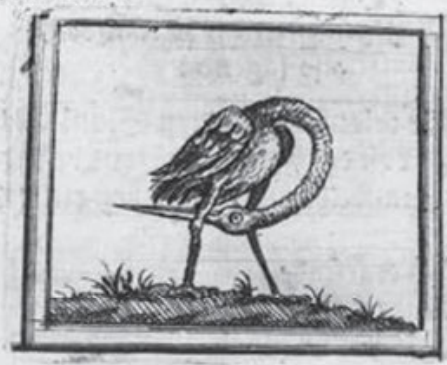

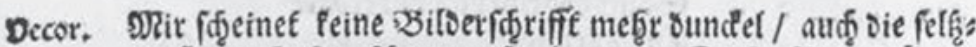
amfte weif id bnfd̨fwer aujzulegen. Diefer $230 \mathrm{get}$ iff Der fdetwarke Stord/ vno bedeutef Den Mercurius/ welder in Der

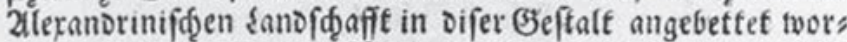
Den. Durḑ Das Eateinifकृe A, tveldges den 2 nfang be deutet / ftellet er vielmefor ber2Bernunffit/als benenSiñen vor/oaj oie

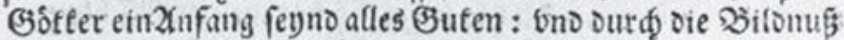

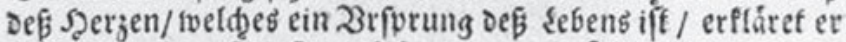

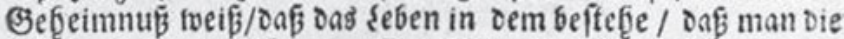

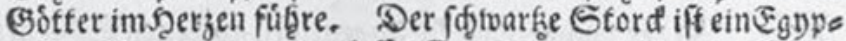
fifder 23ogel / but verlaffet Egypten niemab̨len. Ein po fditwades కfoier leforet ben \$?enfiden/ toie er fein 23atfertand lieben folle. Ees ift ein Feind ber Sọlangen / verjaget/bets

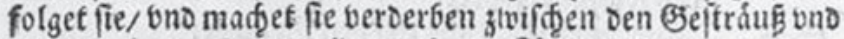

- Dornbeden. Difez follen aud die Rónige tøan / und die Sthlangen verjagen.

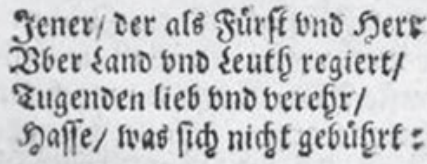

mir.

Abb. 6a: Schwarzer Storch, Holzschnitt. In: Niccolò Minato (Text) und Antonio Draghi (Musik): Die Egyptischen Piramiden Oder Feuer-Säulen [...]. Wien 1697, Fol. 2, Wolfenbüttel, Herzog August Bibliothek, http://diglib.hab.de/drucke/xb-8671/start.htm, 12. September 2016. 


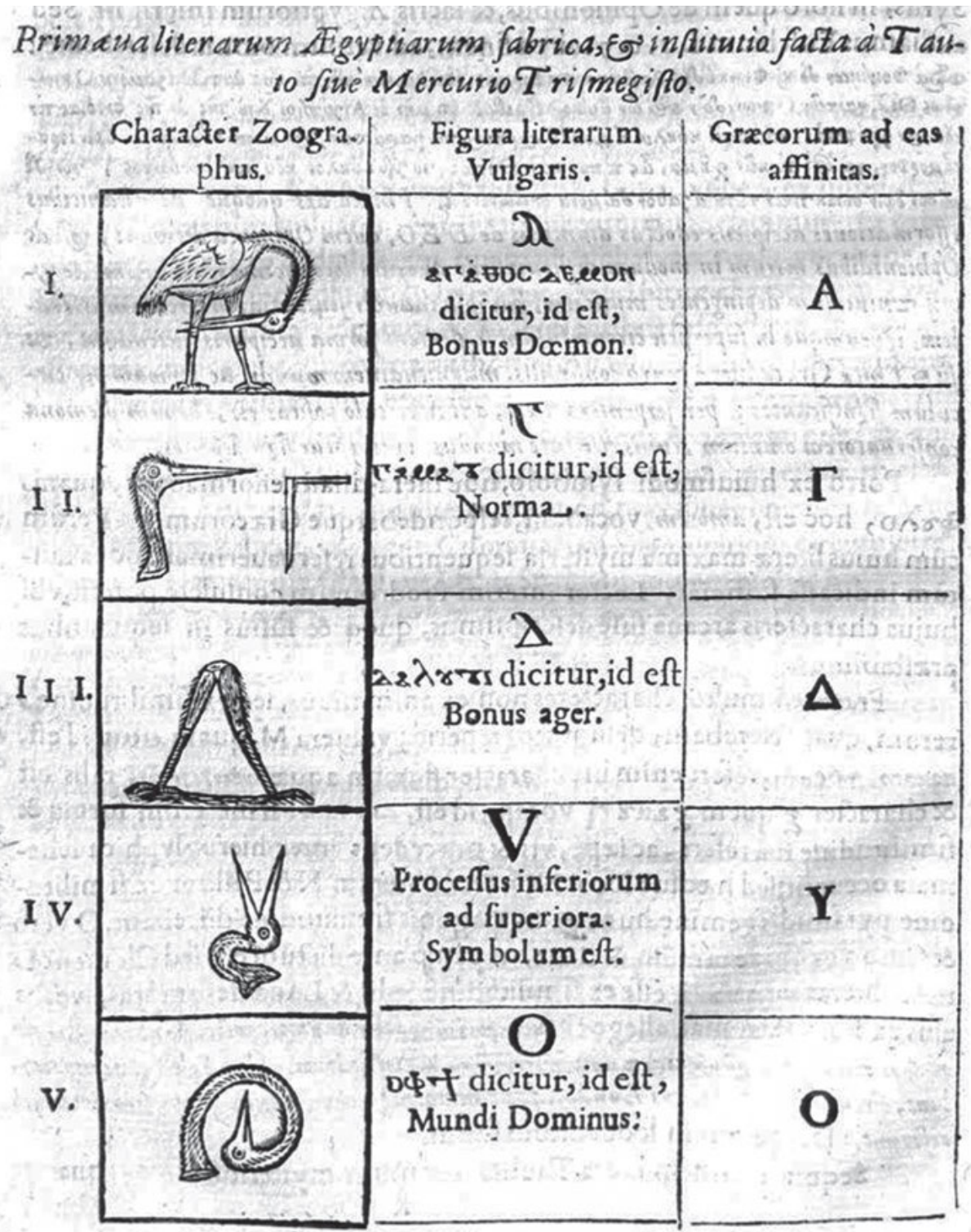

Abb. 6b: Schwarzer Storch. In: Athanasius Kircher, Turris Babel, sive Archontologia qua primo Priscorum post diluvium bominum vita, mores rerumque gestarum magnitudo [...], Buch 3, Linguarum divisio et transmigrationis Gentium Historia continetur, Kapitel 9, Sektion 1, Amsterdam 1679, 177-178, Heidelberger Bestände digital http://digi.ub.uni-heidelberg.de/diglit/kircher1679/0205, 16. September 2016. 
dem ebenbürtige Feinde einander gegenübergestellt worden waren. Caesar, der Inbegriff des sieghaften Helden, brüstet sich Juba gegenüber seiner für das Vaterland vollbrachten Taten und besingt die von ihm vollbrachte gerechte Rache und die von ihm geschossenen Pfeile in einer virtuosen Arie mit einem zwei Oktaven umfassenden Ambitus, als solle musikalisch vorgeführt werden, welchen Raum Caesar einzunehmen imstande ist. Juba antwortet mit einer melismenreichen, verängstigten Arie: Er hat im Krieg die Stärke (fortezza) des Caesarischen Arms (braccio) kennengelernt und spürt diese real an seinen Fesseln (laccio). Das Wertgefälle, die Wert-Unwert-Opposition zwischen Christen und Heiden, zwischen Europäern und (edlen),Wilden ${ }^{54}$, wie von diesen beiden Arien markiert, wird noch deutlicher, nimmt man Caesars Arie hinzu. Auf ungewöhnliche Weise überlagern sich darin zwei Formprinzipien, Da-Capo-Arie und Chaconne auf ein viertaktiges fallendes Bassmodell, über dem zwei Soloviolinen virtuos diminuieren. Musikalische Mittel (Artifizialität, Beständigkeit im Bass, aber auch die Anspielung auf das Tanzlied) werden hier in den Dienst der Zeichnung eines vielschichtigen kaiserlichen Charakters genommen.

Habsburgische Stärke und mauretanische Unterlegenheit werden damit musikalisch vernehmbar - nicht im Sinne von musikalischem Exotismus, sondern im Sinne einer musikalischen Personencharakterisierung. Damit ist um die Jahrhundertwende ein erster Schritt getan hin zum musikalisch Hörbarwerden des Anderen, anfangs vor allem einer Musik alla turca, die Sache des 18. Jahrhunderts wurde.

\section{Abbildungsnachreise}

$\begin{array}{ll}\text { Abb. 1: } & \text { (c) Österreichische Nationalbibliothek, Wien. } \\ \text { Abb. 2: } & \text { (C) Herzog August Bibliothek, Wolfenbüttel. }\end{array}$

Abb. 3: $\quad$ Bernhard Klein: Randfiguren. Othello, Oroonoko und die kartographische Repräsentation Afrikas. In: Ina Schabert und Michaela Boenke (Hg.): Imaginationen des Anderen im 16. und 17. Jahrbundert (Wolfenbütteler Forschungen; 97). Wiesbaden 2002, 215, Abb. 7.

Abb. 4, 5 und 6a: (C) Herzog August Bibliothek, Wolfenbüttel.

Abb. 6b:

(C) Heidelberger Bestände digital.

54 Vgl. Peter Strohschneider: Fremde in der Vormoderne. Über Negierbarkeitsverluste und Unbekanntheitsgewinne. In: Anja Becker und Jan Mohr (Hg.): Alterität als Leitkonzept für historisches Interpretieren (Deutsche Literatur. Studien und Quellen; 8). Berlin 2012, 387-416. 